\title{
Diabetes in pregnancy among Sri Lankan women: gestational or pre-gestational?
}

\author{
K G H Jayathilaka', S Dahanayake', R Abewardhana ${ }^{2}$, A K P Ranaweera ${ }^{3}$, M R M Rishard ${ }^{3}$, C N \\ Wijeyaratne ${ }^{4}$
}

Sri Lanka Journal of Diabetes Endocrinology and Metabolism 2011; 1: 8-13

(Index words: pregnancy, diabetes, gestational, pre-gestational)

\begin{abstract}
Introduction: There is an exponential rise in the occurrence of diabetes during pregnancy in South Asia. However data is sparse on the actual pre-gestational diabetes mellitus (PGDM) versus gestational diabetes mellitus (GDM) case-mix. The applicability of the WHO gold standard diagnostic tool $-75 \mathrm{~g}$ oral glucose tolerance test (OGTT) - and its optimal timing between 24-28 weeks gestation in South Asians is unknown.
\end{abstract}

Objective: To assess optimal timing for diagnosis, determine the case-mix of PGDM and GDM and their specific risk profiles, insulin needs and pregnancy outcomes among Sri Lankans.

Method: Prospective data was collected from consecutive women diagnosed with diabetes in pregnancy, at the Professorial Unit, De Soysa Hospital, Colombo from 1st January 2010, - 28th Feb 2011. All were screened by an initial 2 hour post prandial (PPBS) at antenatal booking and risk stratified to determine the optimal timing of OGTT.

Results: (Total $n=140$ ) GDM and PGDM occurred in $82 \%$ and $18 \%$ of patients respectively.

GDM ( $n=115)$ Mean age 32.16 \pm 5.26 ; booking POA $13.7 \pm 5.8$ weeks; booking BMI $26 \pm 4.9 \mathrm{~kg} / \mathrm{m}^{2}$. Risk factor profile $-1(33 \%) ; 2(29.3 \%) ; \geq 3(29 \%) ; 64 \%$ were detected before 24 weeks. Those $>30$ years were $67 \%$ among early diagnosis versus. $36 \%$ among those diagnosed between $24-28$ weeks $(p=0.02)$. Previous miscarriages were $36 \%$ among early diagnosed versus. $18 \%$ among those diagnosed late $(p=0.145)$. Pregnancy induced hypertension occurred in $7.8 \%$ with similar occurrence in both subgroups.

Pregnancy outcome was similar in the two subgroups ( $100 \%$ live births, mean birth weight $3.127 \pm 0.50 \mathrm{~kg}$, macrosomia $21 \%$; LSCS $43 \%$, pre-term $6.9 \%$; neonatal hypoglycaemia and jaundice $11 \%$; congenital malformation $=1(0.9 \%)$.

Pre-GDM $(n=25)$ Mean age 32.92 $\pm 5.9(2 / 3>30$ years); booking POA $12.7 \pm 6.1$ weeks; booking BMI $23.49 \pm 3.52 \mathrm{~kg} / \mathrm{m}^{2}$, significantly less than GDM group ( $\left.p=0.03\right)$. Risk factor profile $-1(28 \%) ; 2(28 \%) ; \geq 3$ $(32 \%)$. Previous miscarriage had occurred in $24 \%$ with more still births than in GDM group $(p=0.002)$. Previous GDM was significantly more $(p=0.03)$. Pregnancy induced hypertension occurred in $8 \%$.

Pregnancy outcome: $100 \%$ live births. Mean birth weight $3.014 \pm 0.56 \mathrm{~kg}$; macrosomia $20 \%$; LSCS $44 \%$; pre-term $16 \%$; neonatal jaundice and hypoglycaemia $20 \%$ (significantly more than GDM group, $\mathrm{p}=0.02)$; congenital malformation $=1(4 \%)$.

Conclusion: Unequivocal PGDM occurs among $18 \%$ of pregnant diabetics, among older multiparous women with previous GDM and still births. GDM was diagnosed before the internationally recommended 24 weeks in $64 \%$, although their insulin requirement was significantly less than those diagnosed after 24 weeks.

Recommendations: 1) The current timing in pregnancy for screening by OGTT in Sri Lanka requires review. 2) A comprehensive pre-conception screening programme, particularly for older women with previous GDM and/or previous pregnancy loss, is required.

${ }^{1}$ Research Coordinator, Department of Obstetrics and Gynaecology, Faculty of Medicine, University of Colombo, ${ }^{2}$ Diabetes Educator Nursing Officer, ${ }^{3}$ Registrar in Obstetrics and Gynaecology, De Soysa Hospital for Women, Colombo, ${ }^{4}$ Professor in Reproductive Medicine, Department of Obstetrics and Gynaecology, Faculty of Medicine, University of Colombo, Sri Lanka and Honorary Consultant Physician, De Soysa Hospital for Women, Colombo, Sri Lanka. 


\section{Introduction}

There is an exponential rise in the prevalence of diabetes throughout the world, with South Asia being its focal point. Its incidence has increased in South Asia by $111 \%$ in the past 15 years, when compared to other continents such as North America, Australia and Europe which have less than a 50\% rise (1). Hence, Sri Lankans are clearly a high risk population. Gestational diabetes mellitus (GDM) is emerging as a common medical complication of pregnancy (2), with a parallel increase to the pandemic of type 2 diabetes mellitus. Currently GDM affects approximately $7 \%$ of all pregnancies and up to $14 \%$ of pregnancies in high-risk populations while pregestational diabetes mellitus (PGDM) is estimated to affect about 1.3\% (3). The incidence of GDM in South India is reported to be $16.55 \%$, while our own incidence in the community was $10.3 \%(4,5)$.

The American Diabetes Association (ADA) defines GDM as "glucose intolerance of any degree with the onset or first recognition during pregnancy, and irrespective of whether or not insulin is required or the condition persists after pregnancy" (6). Therefore pregnancy can be perceived as a "stress test" for glucose intolerance and a predictor of future diabetes/pre-diabetes in any given population. Diabetes in pregnancy, both GDM and pregestational diabetes mellitus (PGDM), are linked to several maternal and fetal/neonatal complications $(7,8,9)$. PGDM carries a greater risk for mother and baby, particularly if poorly controlled prior to a planned pregnancy. There is no reliable data on the actual pre-gestational diabetes mellitus (PGDM) versus gestational diabetes mellitus (GDM) case-mix in Sri Lanka. A formal pre-conception assessment package to screen for diabetes mellitus is yet not in place. Neither is the suitability of the diagnostic tool (the 75g oral glucose tolerance test - OGTT) to be performed in South Asian women at the recommended 2428 weeks of gestation clearly known.

\section{Objectives}

We proceeded to determine the case-mix of PGDM and GDM, specific risk profiles, insulin requirement and pregnancy outcomes and to assess the optimal timing for diagnosis by OGTT in a cohort of pregnant Sri Lankan women with diabetes attending a single tertiary care unit in Colombo, Sri Lanka.

\section{Method}

This is a prospective review of 140 diabetic pregnant women attending the antenatal clinic conducted by Professorial Unit of De Soysa Hospital for Women. Consecutive women with abnormal glucose tolerance, who attended the clinic between January 2010 and February 2011, were recruited.
The database was maintained by pre-intern medical graduates, by using a previously validated intervieweradministered questionnaire. The information was gathered during the patients' antenatal clinic visits and hospital admissions, through a one to one in-depth interview and reliability of clinical information determined by cross checking past medical records of each subject. Ethical approval was granted by the Ethics Review Committee of the Faculty of Medicine, University of Colombo to maintain a database.

We defined pre-gestational diabetes mellitus (PGDM) as abnormal glucose tolerance recognized prior to conception, which the patient was aware of at the time of her antenatal booking visit in the first trimester. We included into the GDM group all patients who revealed no previous history of diabetes mellitus but were diagnosed by the attending physician (CNW) based on WHO criteria for diagnosing abnormal glucose tolerance by a $75 \mathrm{~g}$ oral glucose tolerance test (OGTT).

This was possible because we adopted an in-house protocol that all women with previously unknown diabetes/pre-diabetes undergo a 2 hour postprandial blood glucose (2hr PPBS) test at antenatal booking in the first trimester. When this value exceeds $120 \mathrm{mg} / \mathrm{dl}$, which is the upper limit of target for normoglycaemia in pregnancy complicated by diabetes, we proceed to performing the OGTT as soon as possible in early pregnancy (well before 24 weeks gestation). If the OGTT is abnormal in early pregnancy and particularly before 24 weeks of period of gestation (POG), we categorize them as with 'early diagnosed' GDM. If the OGTT thus performed as soon as the $2 \mathrm{hr}$ PPBS shows a result $>120 \mathrm{mg} / \mathrm{dl}$ proves to be normal, we repeat the OGTT in later pregnancy at the recommended period of gestation (24-28 weeks) and if found to be abnormal categorize them as 'late diagnosed' GDM.

We identified the standard risk factors for GDM in all women to risk stratify the pregnant women. These include the booking visit and first trimester BMI $\geq 25 \mathrm{~kg} / \mathrm{m}^{2}$ recognized as maternal obesity, maternal age $\geq 35$ years, polycystic ovary syndrome (PCOS), family history of diabetes mellitus in first degree relatives, migrant workers, previous fetal macrosomia ( $>3.5 \mathrm{~kg}$ at term pregnancy), previous fetal loss as still birth or late spontaneous miscarriage, recurrent pregnancy induced hypertension particularly of the gestational non proteinuric variety, medications with steroids or anti psychotics and excessive weight gain in the current pregnancy (10). We also adopted a standard clinical approach that despite a "normal" $2 \mathrm{hr}$ PPBS value at antenatal booking in very early pregnancy, in the presence of multiple risk factors $(\geq 2)$ that there is compelling evidence of a higher risk for diabetes and ensured that the OGTT is performed "as early as possible" in the high risk women rather than awaiting the recommended 24-28 weeks of gestation. 
All those with abnormal OGTT were initially managed by intensive dietary modifications with blood glucose monitoring, with institution of insulin therapy as deemed appropriate by the glycaemic profile. Blood sugar control was assessed by serial blood sugar series over 24 hours. Adjustment of the dose of insulin was made by the attending physician to achieve target blood glucose values of premeal $70-90 \mathrm{mg} / \mathrm{dl}$ and $2 \mathrm{hr}$ PPBS $<120 \mathrm{mg} / \mathrm{dl}$ as early as possible and aimed at being achieved throughout pregnancy by fortnightly review. All those on insulin therapy and with additional risks were admitted at 38 weeks gestation to plan the mode and timing of delivery, while those on dietary modification alone were assessed individually to deliver before 40 weeks. Demographic data, past obstetrics history, current pregnancy factors and associated complications, risk factors for GDM, serial fetal assessment by ultrasound scan, biochemical testing, insulin dose required, mode and timing of delivery, birth weight, pregnancy outcome in respect of maternal, and perinatal complications were carefully recorded and rechecked.

\section{Statistical analysis}

Data analysis was performed using SPSS13 $\mathbb{R}$ software. Mean value and standard deviation was estimated for each continuous variable, such as maternal age, booking BMI, POA of booking visit, birth weight; while proportions by percentages were estimated for categorical variables such as sub groups of early and late diagnosed GDM, dietary modification alone and insulin treated groups, the presence and number of risk factors, mode and timing of delivery, maternal, fetal and neonatal complications.

Chi square value was used to compare frequency and/or proportions while Student's t test was used to compare continuous variables. $\mathrm{P}<0.05$ was considered as the level of significance.

\section{Results}

Among a total of 140 women studied during this period, GDM occurred in $115(82 \%)$ with PGDM in $25(18 \%)$.

Table 1. Comparison demographic data, risk factors, pregnancy outcome and neonatal complications of gestational versus pre-gestational diabetes subgroups

\begin{tabular}{|c|c|c|c|}
\hline Characteristic & $G D M N=115$ & $P G D M N=25$ & $P$ value \\
\hline Age (years) & $32.16 \pm 5.26$ & $32.92 \pm 5.9$ & NS \\
\hline Booking POA weeks & $13.7 \pm 5.8 \mathrm{~s}$ & $12.7 \pm 6.1$ & NS \\
\hline $\operatorname{BMI}\left(\mathrm{kg} / \mathrm{m}^{2}\right)$ & $26 \pm 4.9$ & $23.49 \pm 3.5$ & 0.034 \\
\hline Parity & $2.44 \pm 1.2$ & $3.08 \pm 1.4$ & 0.023 \\
\hline \multicolumn{4}{|l|}{ Risk Factor } \\
\hline $\mathrm{BMI}>25 \mathrm{~kg} / \mathrm{m}^{2}$ & $55(47 \%)$ & $5(20 \%)$ & 0.037 \\
\hline Age $>30$ yrs & $71(61.7 \%)$ & $19(76 \%)$ & NS \\
\hline Previous birth wt $>3.5 \mathrm{~kg}$ & $15(13.0 \%)$ & $4(16 \%)$ & NS \\
\hline Previous IUD & $4(3.5 \%)$ & $5(20 \%)$ & 0.002 \\
\hline Previous miscarriage & $31(26.3 \%)$ & $6(24 \%)$ & NS \\
\hline Family T2DM & $50(43.5 \%)$ & $15(60 \%)$ & NS \\
\hline Previous GDM & $23(20 \%)$ & $10(40 \%)$ & 0.033 \\
\hline PCOS & $6(5.2 \%)$ & $1(4 \%)$ & NS \\
\hline \multicolumn{4}{|l|}{ Pregnancy outcome } \\
\hline Live births & $100 \%$ & $100 \%$ & - \\
\hline Operative delivery & $43 \%$ & $44 \%$ & NS \\
\hline Preterm delivery & $6.9 \%$ & $16 \%$ & NS \\
\hline Neonatal complications & $14.7 \%$ & $40 \%$ & 0.012 \\
\hline Maternal sepsis & $2.6 \%$ & $16 \%$ & 0.005 \\
\hline PIH detected & $7.8 \%$ & $8 \%$ & NS \\
\hline Birth weight (kg) & $3.127 \pm 0.50$ & $3.014 \pm 0.56$ & NS \\
\hline Macrosomia & $21 \%$ & $20 \%$ & NS \\
\hline Congenital anomalies & $0.9 \%$ & $4 \%$ & \\
\hline \multicolumn{4}{|l|}{ Neonatal complications } \\
\hline Jaundice & 8 & 6 & 0.01 \\
\hline Hypoglycaemia & 7 & 3 & 0.295 \\
\hline Low birth weight & 1 & 1 & \\
\hline Prematurity & - & 1 & \\
\hline Birth asphyxia & 1 & - & \\
\hline
\end{tabular}

$(\mathrm{NS}=$ not significant, $\mathrm{BMI}=$ body mass index, $\mathrm{T} 2 \mathrm{Dm}=$ type 2 diabetes mellitus, $\mathrm{IUD}=$ intrauterine death, $\mathrm{PCOS}=$ polycystic ovary syndrome, GDM = gestational diabetes, $\mathrm{PIH}=$ pregnancy induced hypertension) 
Table 2. Pregnancy outcomes in early and late diagnosis groups among those with gestational diabetes (GDM)

\begin{tabular}{llll}
\hline Characteristic & Early diagnosed GDM & Late diagnosed GDM & $P$ value \\
\hline Birth weight & $3.25 \pm 0.07 \mathrm{~kg} / \mathrm{m}^{2}$ & $2.91 \pm 0.11 \mathrm{~kg} / \mathrm{m}^{2}$ & 0.012 \\
Shoulder length & $37.3 \pm 2.7 \mathrm{~cm}$ & $36.4 \pm 2.8 \mathrm{~cm}$ & $\mathrm{NS}$ \\
Preterm delivery & $15 \%$ & $9 \%$ & $\mathrm{NS}$ \\
LSCS- & & & \\
Elective & $30.7 \%$ & $27.3 \%$ & $\mathrm{NS}$ \\
Emergency & $10 \%$ & $9 \%$ & \\
Neonatal, complications & $23 \%$ & $18 \%$ & $\mathrm{NS}$ \\
Maternal complications & $5.1 \%$ & $4.5 \%$ & $\mathrm{NS}$ \\
Congenital abnormality & 1 & - & \\
\hline
\end{tabular}

Their demographic characteristics, risk factor profiles, pregnancy outcomes are depicted in Table 1. Both groups of women are of similar age and period of gestation at antenatal booking in the first trimester. However the PGDM group had a significantly lower BMI and higher parity. The GDM group when further sub divided into two groups depending on the period of gestation (POG) of diagnosis, as before 24 weeks categorized as 'early' diagnosis and after the recommended 24 weeks as 'late' diagnosis, show that those diagnosed early comprised $64 \%$ of the group. Both these subgroups had a similar parity of 2, while women older than 30 years was significantly more (67\%) among those diagnosed 'early' $(p=0.02)$. Both groups had similar BMI at antenatal booking $26.10 \pm 3.7$ versus. $24.97 \pm 6.07 \mathrm{~kg} / \mathrm{m}^{2}(\mathrm{p}>0.05)$ (Table 2).

Significant risk factors identified included maternal age $>35$ years, past history of miscarriage or still births, previous birth weight $>3.5 \mathrm{~kg}$, family history of diabetes mellitus in first degree relative(s), past history of GDM or $\mathrm{PIH}$, previous features of PCOS and current medication with steroids or antipsychotics. Although at least one risk factor was evident in $62 \%$ diagnosed 'late' versus $34 \%$ in those diagnosed 'early', the presence of 2 or more risk factors was significantly greater in those found to have 'early' GDM ( $\mathrm{p}=0.04$ ) ( 2 risk factors $37 \%$ vs. $22 \%$ and $\geq 3$ risk factors in $29 \%$ vs. $17 \%$ ). Comparison of individual risk factors revealed that a previous history of GDM was significantly more in the 'early' GDM group $(p=0.03)$. The only baby born with a congenital abnormality was to a para 2 woman aged 35 years, with a BMI of $32.8 \mathrm{~kg} / \mathrm{m}^{2}$, who had her ante-natal booking at 18 weeks and categorized as early GDM. She was a diagnosed patient with PCOS and had previous history of GDM. Despite dietary modification and achieving good glycaemic control she gave birth to a term, live baby, weighing $3.654 \mathrm{~kg}$, with phocomelia by caesarean delivery.

\section{Discussion}

This tertiary clinic based urban cohort of pregnant diabetics reveals for the first time in Sri Lanka that the ratio of pre-gestational to gestational diabetes is approximately $1: 4$, which is a remarkably high ratio for women in their early $30 \mathrm{~s}$. In view of the mean period of gestation at ante-natal booking of the pre-gestational diabetics being at the completion of the period of organogenesis, and the four-fold greater occurrence of congenital fetal malformations in them, this clinic based data further highlights the deficiencies in the current health care delivery system for diabetes care in women of reproductive age. As suggested by others (11), this study highlights the need for a simple low-cost pre-conception package to be made available for all diabetic women of reproductive age attending primary care and tertiary care services and the need to adopt a comprehensive family planning counselling service in the diabetes care for women.

Although there is a limitation in the selection of this clinic based sample, which is more liable to selection bias, it reveals the actual patient characteristics of a busy urban setting clinical service that also brings into question the applicability of the recommended timing of the OGTT for diagnosing diabetes in pregnancy. There is a high probability that those with 'early' GDM we identified were more likely to have had previously undetected pregestational abnormal glucose tolerance. The community survey conducted in 2003/4 in a semi-urban Colombo based sample revealed that GDM occurred in $10 \%$ of the community (5). In the light of our current findings of the early detection of GDM in this hospital based sample, we need to seriously question the current screening strategy for GDM in the community maternity and child health $(\mathrm{MCH})$ service. Although the ADA recommends any woman with an "average risk" be screened at 24-28 weeks of POA (12), the current data confirms that $66 \%$ of the 
women diagnosed with GDM before 24 weeks had at least two standard risk factors. This argues for the need for universal screening of Sri Lankan pregnant women irrespective of the number of risk factors, and those without proper pre-conception assessment to be screened in the first trimester. The need to screen early for GDM by the OGTT must be dependent on the presence of a high risk status, absence of pre-conception assessment whilst also taking into account the clear ethnic risk for diabetes in our community $(13,14$, and 15). A larger scale case control study is recommended to identify the optimal timing for screening (16). Based on our findings, it can be extrapolated that there is an increase in the prevalence of preexisting diabetes in our population, particularly among younger women early in their reproductive years. These issues require appropriate consideration when planning re-organization of the current health service delivery.

It is noteworthy, that despite no significant difference in the individual risk factors, a past history of GDM was significantly higher among those with early diagnosed GDM. This supports our hypothesis of the high probability for a larger proportion of women in the early diagnosed group of GDM to have had previously undetected chronic diabetes / pre-diabetes as many groups including us have shown that nearly $40-60 \%$ of those with previous GDM progress into chronic diabetes and the metabolic syndrome, as early as 3 years post partum $(7,17,18,19)$. It is also interesting that those with clear PGDM in this study had a significantly lower BMI. The absence of data collection of the waist circumference in the 1 st trimester affects a clear conclusion or interpreting this finding.

As concluded previously universal screening is the most sensitive strategy in identifying nearly all women with GDM. Because of their high risk of type 2 diabetes later in their life, the opportunity to provide counselling on early lifestyle modification will be missed by not having an effective follow up programme for women with previous GDM. No doubt the accurate and timely diagnosis of GDM will also impact in the short term on pregnancy outcome. The responsibility of long-term follow up for these young women at metabolic risk no doubt falls on our primary care services. This is well supported by the fact that $40 \%$ of PGDM women had a past history of GDM. Hence, we recommend that until more reliable evidence is available all women with probable PGDM due to early diagnosis of GDM be encouraged long term follow up to achieve metabolic risk modification and regular screening for metabolic disease (20). This strategy would be in keeping with early initiation of primary prevention of diabetes and its associated medical problems. Furthermore, this will also ensure improved pre-conception assessment and better metabolic status for their future pregnancies.

Our data also confirms that the risk of neonatal as well as maternal complications being significantly more in the pre-gestational diabetic woman; while those with early diagnosis of GDM appear to follow a similar trend. Moreover the significantly greater birth weight in the group with 'early' GDM than those diagnosed at the recommended period of gestation supports our hypothesis that the early diagnosed group possibly falls within a more severe category of pre-pregnant metabolic disease. Albeit a small tertiary hospital based sample, this data provides valuable information to encourage a more detailed assessment of previous pregnancy outcomes and in particular birth weight exceeding $3.5 \mathrm{~kg}$ (95th centile of mean birth weight for Sri Lanka), intrauterine deaths and mid trimester miscarriages in young women to help risk stratify them into regular screening programmes for Sri Lanka. The fact that both PGDM and GDM groups had similar occurrence of pregnancy induced hypertension also supports the need for a multiple risk factor approach to the problem of chronic non-communicable disease being adopted in our community health programme, which must also include such young high-risk women.

To summarize, the case mix of diabetes in pregnancy in an urban based tertiary clinic in Sri Lanka confirms a gestational to pre-gestational ratio of 1: 4 among women in their early $30 \mathrm{~s}$. Their mean period of gestation at antenatal booking was well after the period of organogenesis. Two thirds of the women with GDM were diagnosed before the recommended period of gestation of 24 weeks, where early screening was necessary due to the presence of 2 or more risk factors in addition to their high ethnic risk. Previous GDM was significantly more in those diagnosed early, who also had a significantly higher birth weight in their current pregnancy. Although the majority of women with early GDM required dietary intervention alone with a smaller proportion requiring insulin than those diagnosed after 24 weeks, the birth weight being higher in the early GDM group requires further study. Women with unequivocal pre-gestational diabetes had more severe neonatal complications in the form of hypoglycaemia and jaundice, more congenital anomalies and a greater incidence of maternal sepsis.

\section{Conclusion}

We conclude that pre-gestational diabetes occurs at least in a fifth of urban based women in Sri Lanka and is associated with higher maternal age, multiparity, previous gestational diabetes and intrauterine deaths with maternal BMI not being an important risk factor. Based on the current data we recommend a more comprehensive preconception screening programme for the older women with previous GDM and/or previous pregnancy losses and a robust programme to ensure long term follow up of women with gestational diabetes after delivery, with a view to prevent progression to frank type 2 diabetes mellitus and metabolic disease. The MCH programme also requires exploring the optimum timing for screening for diabetes in pregnancy by OGTT in the current context. 


\section{References}

1. Zimmet P. Globalization, coca-colonization and the chronic disease epidemic: can the doomsday scenario be averted? J Intern Med 2000; 247: 301-10.

2. Wijeyaratne CN, Arandara D, Gunawardane PTK, Jayawardane DBIA, Randeniya, Seneviratne HR. Gestational diabetes mellitus; the Sri Lankan perspective: Sri Lanka Journal of Obstetrics and Gynaecology 2006; 28: $12-8$.

3. American Diabetes Association. Gestational diabetes mellitus. Diabetes Care 2004; 27(Suppl. 1): S88-S90.

4. Seshiah V, Das AK, Balaji V, et al. Diabetes in Pregnancy Study Group. Gestational diabetes mellitus-guidelines. $J$ Assoc Physicians India 2006; 54: 622-8.

5. Ginige S, Wijewardhana K, Wijeyaratne CN. Prevalence of gestational diabetes mellitus in Homagama Divisional Director of Health Services Area. Journal of the College of Community Physicians of Sri Lanka 2004; 9: 40-2.

6. American Diabetes Association. Gestation diabetes mellitus (position statement). Diabetes Care 2004; 27: 88-90.

7. Mallah KOE, Narchi H, Kulaylat NA, Shaban MS. Gestational and pre-gestational diabetes: comparison of maternal and fetal characteristics and outcome. International Journal of Gynecology and Obstetrics 1997; 58(2): 203-9.

8. Mitanchez D. Foetal and neonatal complications in gestational diabetes: perinatal mortality, congenital malformations, macrosomia, shoulder dystocia, birth injuries, neonatal complications. Diabetes Metabolism 2010; 36: 617-27.

9. Rosenn B, Miodovnik M, Combs CA, et al. Glycaemic thresholds for spontaneous abortion and congenital malformations in insulin-dependent diabetes mellitus. JObstet and Gynecology 1994; 84: 515.

10. Metzger BE, Buchanan TA, Coustan DR et al. Summary and Recommendations of the Fifth International Workshop-
Conference on Gestational Diabetes Mellitus. Diabetes Care 2007; 30: S251-60.

11. Wahabi HA, et al. Preconception care for diabetic women for improving maternal and fetal outcomes: a systematic review and meta-analysis. BioMed Central Pregnancy Childbirth 2010; 10: 63.

12. American Diabetes Association: Standards of medical care in diabetes 2011. Diabetes Care 2011;34: S11-61.

13. Shai I, Jiang R, Manson JE, et al. Ethnicity, obesity, and risk of type 2 diabetes in women - a 20 -year follow up study. Diabetes Care 2006; 29:1585-90.

14. Berkowitz GS, Lapinski RH, Wein R, Lee D. Race/ethnicity and other risk factors for gestational diabetes. Am J of Epidemiology 1992; 135: 965-73.

15. Lawrence JM, Contreras R, Chen W, Sacks DA. Trends in the prevalence of preexisting diabetes and gestational diabetes mellitus among a racially/ethnically diverse population of pregnant women 1999-2005. Diabetes Care 2008; 31: 899-904.

16. Wijeyaratne $\mathrm{CN}$, Ginige, Arasalingam A, Egodage C, Wijewardhena K. Screening for gestational diabetes mellitus: the Sri Lankan experience. Ceylon Medical Journal 2006; 51: 53-8.

17. O'Sullivan JB. Diabetes after GDM. Diabetes 1991; 40: $131-5$.

18. Lauenborg J, Hansen T, Jensen DM, et al. Increasing incidence of diabetes after gestational diabetes. Diabetes Care 2004; 27: 1194-9.

19. Wijeyaratne CN, Waduge R, Arandara D, et al. Metabolic and polycystic ovary syndromes in indigenous South Asian women with previous gestational diabetes mellitus. BJOG 2006; 113: 1182-7.

20. Kitzmiller JL, Dang-Kilduff L, Taslimi MM. Gestational diabetes after delivery. Short-term management and longterm risks. Diabetes Care 2007; 30: S225-35. 